\section{REVISTA}

Actualidades Investigativas en Educación

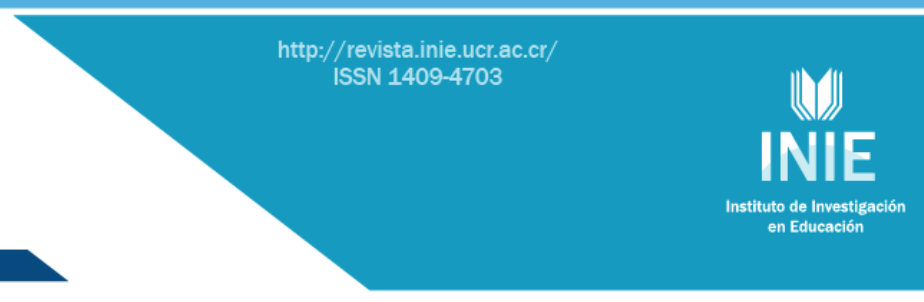

\title{
APRENDIZAJE Y RENDIMIENTO ACADÉMICO EN EDUCACIÓN SUPERIOR: UN ESTUDIO COMPARADO
}

LEARNING AND ACADEMIC ACHIEVEMENT IN HIGHER EDUCATION: A COMPARATIVE STUDY

\section{Volumen 15, Número 3}

Setiembre - Diciembre

pp.1-26

\section{Este número se publicó el $1^{\circ}$ de setiembre de 2015 \\ DOI: http://dx.doi.org/10.15517/aie.v15i3.21072}

Fernando García Gajardo Gonzalo Fonseca Grandón

Lisbeth Concha Gfell

Revista indizada en REDALYC, $\underline{\text { SCIELO }}$

Revista distribuida en las bases de datos:

CATÁLOGO DE LATINDEX, IRESIE, CLASE, DIALNET, DOAJ, E-REVIST@S, SHERPA/ROMEO, QUALIS, MIAR

Revista registrada en los directorios:

ULRICH'S, REDIE, RINACE, OEI, MAESTROTECA, PREAL, CLACSO 


\title{
APRENDIZAJE Y RENDIMIENTO ACADÉMICO EN EDUCACIÓN SUPERIOR: UN ESTUDIO COMPARADO \\ LEARNING AND ACADEMIC ACHIEVEMENT IN HIGHER EDUCATION: A COMPARATIVE STUDY
}

\author{
Fernando García Gajardo ${ }^{1}$ \\ Gonzalo Fonseca Grandón ${ }^{2}$ \\ Lisbeth Concha Gfell
}

\begin{abstract}
Resumen: El presente artículo expone el resultado de dos investigaciones desarrolladas con el propósito de determinar el repertorio de estrategias de aprendizaje (EA) y su relación con el avance curricular y el rendimiento académico de la población estudiantil de dos instituciones de Educación Superior Chilenas, ubicadas en la V y VIII región del país. En ambos casos se aplicó el test de Adquisición, Codificación, Recuperación y Apoyo al Procesamiento de la Información (ACRA). El primer caso corresponde a jóvenes de una carrera de formación de docentes en Educación Primaria y el segundo, a estudiantes pertenecientes a una carrera de nivel técnico, de una institución de las Fuerzas Armadas chilenas. La muestra fue de 342 estudiantes, a quienes se les administró el test entre los años 2010 y 2014. Los hallazgos del estudio develaron que, en general, ambos grupos al ser medidos, contaban con un repertorio de EA escasas. Por otra parte, en el supuesto de que las EA aumentaban mientras se avanzaba en la formación curricular (en este caso de profesores de Primaria), las evidencias arrojaron que ello no ocurre y que estas, se mantienen estables a lo largo de dicho proceso. Finalmente, se corroboró lo que también, en otros trabajos de investigación se ha descubierto, en cuanto a que los estudiantes que tienen un buen rendimiento poseen un repertorio mayor de EA que los que logran un menor rendimiento.
\end{abstract}

Palabras clave: ESTRATEGIAS DE APRENDIZAJE, EVALUACIÓN DE APRENDIZAJES, METODOLOGÍAS DE ENSEÑANZA, EDUCACIÓN SUPERIOR, CHILE

\begin{abstract}
This article presents the results of two researches carried out with a view to determining the repertoire of learning strategies and their association with curricular progress and academic performance of learners from two higher education institutions located in the V Valparaiso region and in the VIII Bio-Bio region. In either case, the ACRA-Abbreviated questionnaire (Acquisition, Codification, Recuperation and Assistance) was applied. The first case corresponds to learners belonging to a study program for the training of primary teachers. The second case corresponds to learners belonging to a vocational and training program of the Chilean Armed Forces. The findings of the study indicate a lower use of teaching and learning strategies in two different groups of students in higher education, as well as little or no increase in learning strategies along the training period. In the end, the findings agreed with other research studies where good or high performace students have a higher repertoire fo strategies than low grade students
\end{abstract}

Keywords: LEARNING STRATEGIES, LEARNING ASSESSMENT, TEACHING METHODOLOGIES, HIGHER EDUCATION, CHILE.

\footnotetext{
${ }^{1}$ Académico de la Universidad Católica de la Santísima Concepción, Chile. Doctor en Diseño Curricular y Evaluación Educativa. Dirección electrónica: fgarcia@ucsc.cl

${ }^{2}$ Académico de la Universidad Católica de la Santísima Concepción, Chile. PhD@ Educación Comparada y Fundamentos de la Educación. Dirección electrónica.gfonseca@udec.cl

${ }^{3}$ Profesora de la Pontificia Universidad Católica de Chile, Magíster en Ciencias de la Educación mención Didáctica e Innovación Pedagógica de la Universidad Católica de la Santísima Concepción, Chile. Dirección electrónica: lisbgfell@gmail.com
}

Artículo recibido: 3 de diciembre, 2014

Enviado a corrección: 27 de febrero, 2015

Aprobado: 17 de agosto, 2015 


\section{Introducción}

Los actuales desafíos demandados a la formación en Educación Superior exigen situar el foco en la evidencia de logro de aprendizaje en estudiantes. Lo anterior, repercute en el rol que asumen docentes y estudiantes en el proceso de enseñanza y aprendizaje (Beltrán, 1998). En este sentido, la preocupación por abordar estos cambios paradigmáticos ha sido objeto de análisis desde múltiples enfoques: conductismo, cognoscitivismo, metacognición, autorregulación, etc. (Mayor, Suengas y González, 1995).

En la visión educativa para la educación del Siglo XXI, destaca el protagonismo estudiantil en el proceso de enseñanza-aprendizaje, considerando de gran importancia el manejo de estrategias de aprendizaje que le permitan enfrentar con éxito los requerimientos de sus estudios y las demandas de la sociedad. En esta dirección, capacidades como la resolución de problemas, trabajo en equipo, emprendimiento, aprendizaje autónomo, formación ciudadana, etc. interpelan los propios procesos intelectuales que asume dicha población.

En este escenario, el enfoque curricular, basado en competencias y resultados de aprendizaje adoptado por las Instituciones de Educación Superior, ha generado amplios espacios para la reflexión en torno a las estrategias de aprendizaje utilizadas por estudiantes. La adopción de este enfoque necesariamente ha implicado cambios en los actores del proceso de enseñanza y aprendizaje. Para el caso de quienes son profesionales de la educación, se espera superar el enfoque de enseñanza basado en la mera transmisión de conocimientos y avanzar hacia el desarrollo de capacidades, habilidades, destrezas y actitudes que permitan disponer de múltiples recursos para actuar competentemente. Por otro lado, la población estudiantil requiere asumir la responsabilidad de su aprendizaje con base en aquellas estrategias que faciliten dicho proceso. En este caso, cada estudiante puede disponer de un repertorio de estrategias que trae desde la educación primaria y secundaria, pero sin duda puede incluir otras que se vean favorecidas como consecuencia de su proceso formativo en la Educación Superior.

Al margen de lo anterior, aún existen tensiones ante un proceso en donde el cuerpo docente sigue fomentando la repetición y reproducción del conocimiento con escaso desarrollo de habilidades en sus estudiantes. De allí, la conveniencia de indagar si efectivamente la población estudiantil posee las "herramientas" para enfrentarse a nuevas formas de aprender, pasando de una posición receptora donde alguien erudito deposita los conocimientos (Freire, 1999) a una protagonista de su aprender. 
A partir de ese planteamiento, se hace necesario indagar si efectivamente un proceso formativo desarrolla o potencia en sus estudiantes nuevas formas de aprender, 0 simplemente mantienen similar repertorio de estrategias a lo largo del proceso de formación. Al parecer el tema de indagar la existencia o manejo de un determinado repertorio de estrategias de aprendizaje queda circunscrito a su existencia o inexistencia y en menor medida a determinar si un proceso formativo logra incrementarlas a lo largo de este.

Diversas investigaciones realizadas en relación con la evaluación de Estrategias de Aprendizaje en estudiantes de Educación Superior permiten otorgar conocimiento acerca de la vinculación de estas con el desarrollo de la inteligencia y la seguridad en sí mismas; el rol que cumplen en una educación centrada en sus estudiantes y por consiguiente el que le compete a cada docente, además, lo referido a la importancia de dichas estrategias en la sociedad del conocimiento (Carbonero, Román y Ferrer, 2013; Correa, Castro y Lira, 2004). De lo anterior, se desprende el interés por indagar acerca del impacto que genera un proceso formativo en el empleo de estrategias a lo largo de los años y si en nuestra realidad socio-cultural, también se logra una relación entre el repertorio de estrategias y el rendimiento académico.

En síntesis, aun cuando en el debate contemporáneo se reconoce la preocupación por las estrategias de aprendizaje de la población de estudiantes de Educación Superior, parece ser que constituye un tema que está más bien a nivel de discurso que efectivamente indagado en la práctica. En este sentido, los estudios se han centrado más bien en discutir cómo se debe actuar bajo un determinado paradigma de aprendizaje, pero escasamente indagan aquellas estrategias utilizadas para aprender por estudiantes de Educación Superior. A partir de allí, surgen interrogantes como: ¿cuáles son las estrategias de aprendizaje que utilizan estudiantes de Educación Superior?; ¿qué relación poseen estas con el avance evidenciado?; ¿cuál es la relación de ellas con el rendimiento académico?.

\section{Referente Teórico}

Para abordar la problemática, objeto de estudio, se hace necesario efectuar un recorrido por el concepto de aprendizaje, planteando las concepciones conductistas, cognitivistas y constructivista y el vínculo con el desarrollo de los procesos cognitivos que se realizan, a través del empleo de estrategias de aprendizaje. A partir de esto teniendo en consideración que el objetivo de toda tarea educativa es el logro de aprendizajes en cualquiera de los ámbitos en los que pueda darse, es clave adecuarse a las necesidades 
presentes y futuras de cada individuo (Pozo y Monereo, 2000) y ciertamente, poseer información acerca de las estrategias de aprendizaje, es un aspecto clave investigar en cualquier proceso de enseñanza.

Dicho proceso de aprendizaje requiere rupturar las clásicas actividades de enseñanza mecanicistas, lo cual demanda a la población estudiantil no solo disponer de recursos estratégicos, sino saber qué, cuándo y cómo utilizarlos con objeto de aprender (Beltrán, 1998; Mayor, Suengas y González, 1995). Dichos recursos -estrategias de aprendizaje- son los que permitirán controlar o seleccionar la información que le dispone aprender contenidos en contextos diversos y experienciales (Díaz-Barriga y Hernández, 2005) y como tal, lo más relevante, aprender a aprenderlos. Esto significa para la Educación Superior, centrarse en el desarrollo de competencias esenciales que permitan a las personas valerse por sí mismas, para intervenir y adaptarse a la sociedad.

Esta capacidad analítica, sumada a una preparación científica, capaz de garantizar una correcta toma de decisiones con el compromiso y responsabilidad por su propio aprendizaje, es clave para favorecer autonomía en los aprendizajes y es estratégico, en cuanto a adquirir las destrezas necesarias para la búsqueda de información, análisis, relación, aplicación y crítica con el fin de reelaborarla en saberes útiles y transformarla en conocimiento.

\subsection{El Aprendizaje}

Partiendo del planteamiento que el aprendizaje es un aspecto clave dentro de la enseñanza, parece prudente considerar el planteamiento que hacen Ausubel, Novak y Hanesin (1997, p. 28) en relación con que "las teorías del aprendizaje son más interdependientes que mutuamente exclusivas", lo cual permite entender la fuerte vinculación o asociación con aspectos teóricos y prácticos para las Ciencias de la Educación y como tal centrado en la praxis educativa. A saber, el concepto de aprendizaje ha estado asociado y centrado en sus inicios con cambios relativamente permanentes en la conducta humana (conductismo), para posteriormente concentrarse en la adquisición de conocimientos o habilidades.

Así es como, según el planteamiento que hacen Mayor, Suengas y González (1995), el concepto de aprendizaje ha pasado desde una concepción conductista a una cognitivista con la incorporación de componentes cognitivos. O cuando se centra la atención en un aprendizaje a partir de los principios constructivistas, planteando que el conocimiento no se adquiere únicamente por interiorización del entorno social, sino que mediante la construcción 
realizada por parte de las personas, en tanto Ausubel y otros (1997) señalan que el aprendizaje significa organización e integración de información en la estructura cognoscitiva, destacando la importancia del conocimiento y la integración de los nuevos contenidos o conocimientos en las estructuras previas del sujeto. Por ello, entonces se entiende que para aprender es necesario relacionar los nuevos aprendizajes con las ideas previas de la población estudiantil, como estructura de acogida, por lo que el aprendizaje es un proceso de contraste, de modificación de los esquemas de conocimiento, de equilibrio, logrando de esta forma que este sea significativo, es decir, real y a largo plazo (Ballester, 2002).

En este sentido, Bruner (2004) indica que el sujeto atiende selectivamente la información, la procesa y organiza, lo cual implica tres procesos: adquisición, transformación y evaluación. Ciertamente, dichos procesos requieren a su vez del manejo de estrategias y técnicas a objeto de favorecer el aprendizaje.

Independientemente de la perspectiva, casi todas las concepciones de aprendizaje han incluido - implícita o explícitamente - tres criterios básicos para su definición: a) el cambio en la conducta de un individuo o su habilidad para hacer algo, b) el cambio como resultado de la práctica o de la experiencia y c) el cambio como un fenómeno que se mantiene de forma perdurable (Puente, 1997).

\subsection{Paradigma del aprender a aprender}

Las demandas desde la sociedad actual implican establecer una nueva cultura del aprendizaje y en coherencia con lo anterior, cambios en la enseñanza universitaria a objeto de que esta responda a la función social de la educación superior, esto es formar profesionales capaces de gestionar conocimientos complejos y hacer más eficaces sus propios procesos de aprendizaje.

En sentido amplio, la educación es una realidad presente en la persona a lo largo de toda su vida, ya sea en ámbitos formales o informales, por lo tanto, lo importante es formar personas preparadas para aprender a lo largo de la vida, pero tan relevante como tener la capacidad de aprender, es necesario desarrollar la actitud de desaprender, la aceptación de la caducidad de los conocimientos y la aceptación del cambio necesario (Pozo y Pérez, 2009).

Esta nueva cultura del aprendizaje requiere que la educación superior se adscriba a un modelo educativo centrado en el aprendizaje, en definitiva, es dicha actividad cognitiva de la población estudiantil, la que le permitirá construir el conocimiento en vez de solamente 
recibirlo cerrado, definido y planificado a manera de educación bancaria (Freire, 1999). Se necesita entonces, transitar desde una educación transmisora de conocimientos a potenciar habilidades superiores y de construcción, a objeto de "desplazar el centro de gravedad tradicional, pasando del conocimiento transmitido hacia uno donde la persona construye y se construye en dicho conocimiento" (Rué, 2009, p.12). Es en este modelo donde cobra valía el que el sistema educativo promueva, impulse y oriente al estudiante en la adquisición de herramientas que le permitan aprender más y con calidad.

\subsection{Teorías del Aprendizaje}

Teniendo en consideración la existencia de diversas teorías que han permitido estudiar, comprender e implementar acciones con el objeto de favorecer aprendizajes, a saber: conductismo, cognoscitivsmo, constructivismo, por nombrar las de mayor impacto. A continuación, se presenta una breve síntesis de cada una de estas teorías.

\subsubsection{Conductismo y aprendizaje}

El aprendizaje, desde esta perspectiva, es definido como algo que puede ser observado y documentado, es decir, hay aprendizaje cuando hay un cambio conductual; en tanto conducta observable e identificable. Este suele ser un cambio relativamente permanente en el comportamiento, el cual refleja adquisición de conocimientos o habilidades y como tal, objetivos potencialmente medibles. En esta teoría, el énfasis está puesto en respuestas mecánicas y repetitivas, sin embargo, no es suficiente concluir que solo a través del reforzamiento se fortalezca una respuesta (Arancibia, Herrera y Strasser, 2005). Para los teóricos conductistas, la conducta a la que siguen consecuencias placenteras tiende a fortalecerse y repetirse y, por lo tanto, se aprende. En cambio, la conducta acompañada de consecuencias desagradables tiende a no repetirse y por ende no se aprende (Henson y Eller, 2000). Estas teorías conductistas del aprendizaje se concentran en acontecimientos externos y el ambiente, para explicar la conducta de la población estudiantil (Pozo, 2005).

\subsubsection{Cognoscitivismo y Aprendizaje}

Al contrario de lo señalado anteriormente, en lugar de dar solo importancia a los elementos externos de la cadena del aprendizaje, la instrucción y la ejecución, se constituyen en eslabones centrales de éste; es decir, cada estudiante da sentido a los 
materiales que procesa y decide lo que tiene que aprender, así como la manera de hacerlo significativo, con el propósito de lograr sus expectativas (Beltrán, 1998).

En términos de Henson y Eller (2000) el proceso de aprendizaje es como un sistema de funciones cerebrales, donde el primer contacto de los estudiantes con la información y el conocimiento, se da a través de los receptores que luego transmiten los datos sobre los estímulos en el ambiente al sistema de procesamiento de la información. Las estructuras en las que tiene lugar el procesamiento de la información son varias cajas negras que representan las fases del proceso, intervienen entre el estímulo de entrada en los registros sensoriales y la salida, traducida en ejecuciones. El acto de comprensión y codificación en la memoria es un proceso asimilativo de construcción de una representación conceptual interna de la entrada, a su vez, la recuperación se concibe como un proceso activo y asimilativo de reconstrucción y no una copia pasiva de lo que está almacenado en la memoria (Flavell, 2000).

Autores como Mayer (2002) plantean ideas como: que el aprendizaje es personal e individual, cada uno construye sus propios significados; a ritmos diferentes y por medios distintos a lo largo de etapas similares de aprendizajes específicos; algo activo e integrativo. Por su parte Ausubel y otros, (1997), señalan que el aprendizaje significativo se produce cuando la nueva información se vincula de manera sustantiva, es decir, no arbitraria con los conocimientos que cada estudiante ya posee, produciéndose una transformación, tanto en el contenido que se asimila como en lo que ya sabe. En otras palabras, que decidida de manera consciente realizar una relación "significativa", entre los nuevos conocimientos y los que ya posee.

\subsubsection{Constructivismo y aprendizaje}

Esta concepción pone el énfasis en los procesos individuales y endógenos de construcción del conocimiento y presenta la actividad autoestructurante. En estas interacciones, ellos despliegan una actividad mental constructiva, encubierta, dirigida a dotar de significado los contenidos académicos. Se aprende cuando se elabora una representación personal sobre un objeto de la realidad o contenido desde las experiencias, intereses y conocimientos previos de los sujetos (Coll, Martín y Mauri, 2007). Coincidiendo con el planteamiento de Pozo (2005) y Pozo y Pérez (2009) se entiende que el aprendizaje desempeña la función de engranaje de los procesos cognitivos al facilitar una amplificación de la capacidad funcional de la memoria de corto plazo, pues todos los procesos cognitivos 
constituyen un sistema de interacción, en que la función dinámica y adaptativa del aprendizaje hace posible la modificación funcional del resto de los procesos. A juicio de ellos cuando la información es aprendida al pie de la letra, sin comprenderla realmente, se tiende fácilmente a olvidar (o a recuperarla con dificultad) respecto de la que se comprende.

El planteamiento que realizan Monereo, Castelló y Clariana (2006) plantea que si a la población estudiantil se le favorece el análisis de las ventajas de un procedimiento sobre otro, en función de las características de la actividad concreta que deben realizar o la reflexión sobre cuándo y por qué es útil aquella técnica o aquel método en cuestión, el proceso se complica y entran en juego las estrategias de aprendizaje para favorecer o ayudar en dicho proceso de aprendizaje.

En definitiva, aprender es buscar repuestas o plantear preguntas relevantes que la población estudiantil ha formulado previamente $y$, no tanto, recibir del docente respuestas detalladas y completas a preguntas que no se han planteado. La tabla 1 permite sintetizar las diferentes concepciones de aprendizaje.

Tabla 1: Concepción de Aprendizaje

\begin{tabular}{|c|c|c|c|c|c|}
\hline Aprendizaje como... & $\begin{array}{l}\text { Conceptos } \\
\text { relevantes }\end{array}$ & $\begin{array}{c}\text { Bajo un } \\
\text { enfoque... }\end{array}$ & $\begin{array}{c}\text { Con una } \\
\text { didáctica } \\
\text { centrada } \\
\text { en... }\end{array}$ & $\begin{array}{l}\text { Años de } \\
\text { inicio }\end{array}$ & $\begin{array}{c}\text { Autores } \\
\text { representativos } \\
\text { /Años trabajos }\end{array}$ \\
\hline $\begin{array}{l}\text { Proceso consistente en } \\
\text { interiorizar o copiar } \\
\text { información. } \\
\text { Adquisición del } \\
\text { conocimiento desde el } \\
\text { exterior. } \\
\text { Como una colección de } \\
\text { datos. } \\
\text { Conductas observables. }\end{array}$ & $\begin{array}{l}\text { Estímulo- } \\
\text { Respuesta- } \\
\text { Refuerzo - } \\
\text { Hábito - } \\
\text { ensayo- } \\
\text { error }\end{array}$ & Asociacionista & $\begin{array}{l}\text { La } \\
\text { memoriza- } \\
\text { ción }\end{array}$ & $\begin{array}{l}\text { A partir de } \\
\text { la década } \\
\text { del } 30\end{array}$ & $\begin{array}{l}\text { Pavlov (1920) } \\
\text { Watson(1913) } \\
\text { Thorndike(1910) } \\
\text { Skinner(1938) }\end{array}$ \\
\hline $\begin{array}{l}\text { Adquisición y } \\
\text { modificación de } \\
\text { estructuras de } \\
\text { conocimiento. } \\
\text { Procesamiento activo de } \\
\text { la información. }\end{array}$ & $\begin{array}{l}\text { Procesos } \\
\text { cognitivos: } \\
\text { La atención, } \\
\text { la memoria, } \\
\text { la } \\
\text { codificación. }\end{array}$ & Cognitivista & $\begin{array}{l}\text { La } \\
\text { comprensión } \\
\text { y la } \\
\text { resolución } \\
\text { de } \\
\text { problemas }\end{array}$ & $\begin{array}{l}\text { Inicio de la } \\
\text { década del } \\
70\end{array}$ & $\begin{array}{l}\text { Piaget (1960) } \\
\text { Ausubel (1963) } \\
\text { Gagné(1965) } \\
\text { Bruner (1966) }\end{array}$ \\
\hline $\begin{array}{l}\text { Construcción del } \\
\text { conocimiento. }\end{array}$ & El sujeto & Constructivismo & $\begin{array}{l}\text { Dar sentido } \\
\text { a las } \\
\text { experiencias } \\
\text { de } \\
\text { aprendizaje }\end{array}$ & $\begin{array}{l}\text { Inicio de la } \\
\text { década del } \\
70\end{array}$ & Vigovsky(1979) \\
\hline
\end{tabular}

Fuente: elaboración propia 


\subsection{Procesos Cognitivos relacionados con las Estrategias de Aprendizaje}

Los procesos cognitivos se entienden como la actividad cerebral encargada de transformar, transportar, reducir, coordinar, recuperar o utilizar una representación mental del mundo (Román y Gallego 2001). En este sentido, el aprendizaje depende de lo que la persona haga, es decir, de los procesos cognitivos que ponga en marcha al aprender $y$, por tanto, de las estrategias que desarrollan esos procesos (Beltrán, 1998).

Román y Gallego (2001) diferencian entre procesos, entendidos como constructos inferidos encargados de transformar, transportar, reducir y coordinar una representación mental del mundo y las estrategias de aprendizaje. Dichas estrategias son denominadas como: Adquisición, Codificación, Recuperación o Evocación y las de Apoyo.

El proceso de adquisición considera las técnicas atencionales y de repetición, las cuales básicamente son las encargadas de seleccionar, transformar y transportar la información desde el ambiente al registro sensorial, desde este a la memoria de corto plazo. Entre las técnicas que favorecen este proceso están: tácticas de subrayado lineal, subrayado idiosincrático y epigrafiado, tácticas para manejar u optimizar los procesos de atención y repetición, el repaso en voz alta, el repaso mental y el repaso reiterado.

El proceso de codificación, es aquel que permite transformar la información desde la memoria de corto a largo plazo. Este proceso implica codificar, lo que representa la comprensión y resignificación del nuevo conocimiento con el propósito de almacenarlo en la memoria de largo plazo. En este sentido, es más fácil aprender y recordar información si se han hecho conexiones de esa información con el conocimiento que ya está en nuestra memoria. La codificación está conformada por tres estrategias de aprendizaje: nemotecnización, elaboración y organización. Cada una de ellas, considera un conjunto de tácticas tales como: acrósticos, metáforas, esquemas, mapas conceptuales y diagramas $\mathrm{V}$, por nombrar algunas, las cuales posibilitan poder efectuar dicho almacenaje.

En relación con el proceso de recuperación o evocación de la información, se encuentran los factores cognitivos que la persona emplea con el propósito de recuperar la información ya procesada y almacenada en la memoria de largo plazo, se conforma por dos estrategias de aprendizaje: la de búsqueda y la de generación de respuesta. Para favorecer dicho proceso se necesita el apoyo de una serie de tácticas tales como: búsqueda de indicios, planificación de respuestas escritas, búsqueda de codificaciones, entre otras.

Finalmente, las estrategias de apoyo al procesamiento de naturaleza metacognitiva y no-cognitiva, son las encargadas de cumplir la función de apoyar o potenciar el proceso de 
adquisición, codificación y recuperación, con lo cual se ve favorecido el buen funcionamiento de todo el sistema cognitivo. Tal como se señaló, estas estrategias se encuentran conformadas por dos métodos de aprendizaje: metacognitivos y socioafectivos. Entre las tácticas de apoyo que se emplean en dicho proceso están, las de planificación, de autoinstrucciones, contradistractoras, de interacción social y las de alto impacto, tales como: la motivación intrínseca y extrínseca.

Complementando lo señalado por Román y Gallego (2001), Cook y Mayer (1983) destacan cuatro procesos esenciales para favorecer aprendizajes:

- Selección: atención selectiva a una parte de la información entrante que pasa a la memoria de trabajo.

- Adquisición: proceso de transferencia de la información desde la memoria de trabajo a la memoria de largo plazo.

- Construcción: proceso de elaboración de materiales informativos, estableciendo conexiones entre las ideas almacenadas en la memoria de trabajo.

- Integración: proceso de búsqueda de conocimientos previos para transformarlos en la memoria de trabajo. Se establecen conexiones externas entre la información entrante y el conocimiento previo.

La tabla 2 permite sintetizar el trabajo desarrollado por Beltrán (1998) y Román y Gallego (2001).

Tabla 2: Procesos Cognitivos

\begin{tabular}{|l|l|lr|}
\hline \multicolumn{1}{|c|}{ AUTORES } & \multicolumn{1}{c|}{ Definición } & \multicolumn{2}{c|}{ Clasificación } \\
\hline Beltrán (1998) & $\begin{array}{l}\text { Sucesos internos que implican una } \\
\text { manipulación de información y son } \\
\text { las metas de las estrategias de }\end{array}$ & $\begin{array}{l}\text { Sensibilización, atención, } \\
\text { adquisición, personalización y } \\
\text { aprendizaje. }\end{array}$ & $\begin{array}{l}\text { control, recuperación, } \\
\text { transferencia y evaluación. }\end{array}$ \\
\hline $\begin{array}{l}\text { Román y Gallego } \\
\text { (2001) }\end{array}$ & $\begin{array}{l}\text { Actividad cerebral encargada de } \\
\text { transformar, transportar, reducir, }\end{array}$ & $\begin{array}{l}\text { Adquisición, } \\
\text { recuperación, rodicicación, }\end{array}$ & apoyor al \\
& coordinar, recuperar o utilizar una & procesamiento & de la \\
información. & la \\
\hline
\end{tabular}

Fuente: elaboración propia en base a Beltrán (1998) y Román y Gallego (2001)

Según Monereo, y otros, (2006) la calidad de los aprendizajes no depende tanto de un supuesto coeficiente intelectual, sino de la posibilidad de captar las exigencias de las tareas en una situación de aprendizaje determinada y controlar con los medios adecuados dicha situación. El interés de lo investigado está referido justamente con los procesos cognitivos y 
metacognitivos que la población estudiantil realiza, y es a través de las estrategias de aprendizaje, la forma en que dichos procesos son llevados a cabo por cuanto son los procesos que sirven de base para la concreción de trabajos intelectuales. Para ello es necesario realizar un procedimiento cognitivo que implique procesar la información, para lo cual es indispensable adquirirla y así, posteriormente, codificarla y recuperarla.

Para Díaz-Barriga y Hernández (2005, p. 234), las estrategias de aprendizaje tienen rasgos comunes, entre ellos destaca que:

- Son procedimientos o secuencia de acciones

- Son actividades conscientes y voluntarias

- Pueden incluir varias técnicas, operaciones o actividades específicas

- Prosiguen un propósito determinado: el aprendizaje y la solución de problemas académicos y /o aquellos aspectos vinculados con ellos

- Son más que los hábitos de estudio porque se realizan flexiblemente

- Pueden ser abiertas (públicas) o encubiertas (privadas)

- Son instrumentos cuya ayuda potencia las actividades de aprendizaje y solución de problemas

- Son instrumentos socioculturales aprendidos en contextos de interacción con alguien que sabe más.

Desde estas características, se establece una aproximación más formal al definir las estrategias de aprendizaje como procedimientos (conjuntos de pasos, operaciones 0 habilidades) que estudiantes emplean en forma consciente, controlada e intencional, como instrumentos flexibles para aprender significativamente y solucionar problemas.

Según Díaz-Barriga, y Hernández, 2005, p. 234), tres son los rasgos más característicos de las estrategias de aprendizaje:

a) La aplicación de las estrategias es controlada y no automática, requiere necesariamente de una toma de decisión, de una actividad previa de planificación y de control de su ejecución. En tal sentido, las estrategias de aprendizaje precisan de la aplicación del conocimiento metacognitivo y sobre todo, autorregulador.

b) La aplicación experta de las estrategias de aprendizaje requiere de una reflexión profunda sobre el modo de emplearlas. Es necesario que se dominen las secuencias de acciones e incluso, las técnicas que las constituyen y que se sepa, además, cómo y cuándo aplicarlas reflexivamente. 
c) La utilización de las estrategias de aprendizaje implica que el estudiante las seleccione inteligentemente de entre varios recursos y capacidades que tenga a su disposición. Se utiliza una actividad estratégica en función de demandas contextuales determinadas y de la consecución de ciertas metas de aprendizaje.

\subsection{Desarrollo de las Estrategias de Aprendizaje}

Para las estrategias cognitivas no existen etapas de desarrollo, algunas de ellas pueden aparecer en etapas tempranas de aprendizaje, y otras en momentos más tardíos del desarrollo, dependiendo del dominio de que se trate y del grado de experiencia de los aprendices en dichos dominios particulares (Díaz-Barriga y Hernández, 2005). Sin embargo, hay un elemento clave que permite mantener un estado mental propicio para el aprendizaje, el cual incluye, entre otras, estrategias para favorecer la motivación y la concentración para reducir la ansiedad, para dirigir la atención a la tarea y para organizar el tiempo de estudio, estas son las estrategias de apoyo (Román y Gallego 2001).

\section{Metodología}

Para el desarrollo de la investigación se formularon los siguientes objetivos:

- Identificar el repertorio de estrategias de aprendizaje que presentan estudiantes de carrera profesional y técnica.

- Determinar la relación existente entre el repertorio de estrategias de aprendizaje y avance curricular en la formación de un profesional de la educación.

- Determinar la relación existente entre el repertorio de estrategias de aprendizaje y el rendimiento académico en la formación de técnicos.

El desarrollo del trabajo investigativo se encuentra adscrito al paradigma cuantitativo por medio de un estudio no experimental, puesto que se privilegió el análisis del fenómeno tal y como se dio en su contexto sin la manipulación o influencia directa sobre las variables analizadas. Además posee un carácter transeccional, ya que los datos se tomaron en un solo momento en cada una de las muestras estudiadas sin considerar la evolución de dichas variables. Específicamente, se utilizó un diseño de tipo descriptivo-correlacional que, por un lado, permitió especificar características relevantes de los grupos de estudiantes incluidos en el estudio y por otro, se establecieron relaciones entre las variables consideradas, 
ajustándose a los criterios propuestos por Hernández, Fernández y Baptista (2010) y Vieytes (2004).

Con el propósito de dar cumplimiento a los objetivos anteriormente explicitados, el trabajo compila dos investigaciones efectuadas por separado, en las cuales se consideró la aplicación del Test de Escalas de Estrategias de Aprendizaje de Román y Gallego (2001). La decisión de utilizar dicho test se vio sustentada por la validez que posee dicho instrumento dado su frecuente empleo en el mundo hispanoparlante. Dicho instrumento, si bien fue creado para aplicar en la educación secundaria, se utiliza también en la universitaria, tanto en contextos naturales de aula como en contextos fuera de ella.

A continuación, se explicitan las variables que están contenidas en el trabajo desarrollado, las cuales se encuentran identificadas y definidas en la Tabla 3:

Tabla 3: Definición de Variables

\begin{tabular}{|l|l|}
\hline \multicolumn{1}{|c|}{ Variables } & \multicolumn{1}{c|}{ Definición } \\
\hline Estrategias de aprendizaje & $\begin{array}{l}\text { Conjunto de actividades, técnicas y medios utilizados por los } \\
\text { estudiantes con el objetivo de facilitar el aprendizaje y la } \\
\text { obtención de óptimos resultados académicos. En su medición } \\
\text { se utilizó el test ACRA el cual mide } 4 \text { escalas independientes } \\
\text { con un total de } 32 \text { técnicas con valores que van entre 1 y } 4\end{array}$ \\
\hline Avance Curricular & $\begin{array}{l}\text { Constituye el nivel de avance en años de formación que } \\
\text { evidencia un estudiante que cursa una carrera de educación } \\
\text { superior en sus estudios. Para ello los datos se agruparon en } \\
\text { torno a cada uno de los cuatro años de dicho proceso, } \\
\text { obtenido a través de la revisión del registro de año de ingreso } \\
\text { de los estudiantes a la carrera cursada. }\end{array}$ \\
\hline Rendimiento Académico & $\begin{array}{l}\text { Capacidad evidenciada por los estudiantes en el logro de los } \\
\text { aprendizajes propuestos las asignaturas de una carrera } \\
\text { técnica de educación superior. En su medición de utilizó una } \\
\text { escala numérica con valores entre } 1 \text { y 10, a partir de la } \\
\text { revisión del expediente institucional de los estudiantes. }\end{array}$ \\
\hline
\end{tabular}

Fuente: elaboración propia

En específico, para el caso de pedagogos en formación, lo que se quiere determinar es el incremento del repertorio de estrategias de aprendizaje a lo largo de una trayectoria académica (años de formación), dado que lo esperable sea que dicha formación permita alcanzar un mayor empleo de las estrategias de aprendizaje.

El otro estudio, coincidente en ambas investigaciones, se refiere a la necesidad de indagar cuáles son las estrategias más fuertemente empleadas por los estudiantes y llegar a comprender la relación con el rendimiento académico que los estudiantes obtienen en determinadas asignaturas de su plan de formación académica. 
La elección de las carreras a las que pertenece la población estudiantil incluida en la muestra se efectuó por medio de un procedimiento no probabilístico e intencionado. Para el caso de quienes estudian la carrera de pedagogía, se optó por una muestra de sujetos voluntarios pertenecientes a una Institución de Educación Superior ubicada en la región del Bio Bío, Chile. A su vez, la muestra de estudiantes pertenecientes a la Escuela Matríz, coincide con la población estudiantil de dicha institución, ubicada también en la misma región de Chile.

Tal como se ha señalado, fue necesario aplicar este instrumento de la forma en que se representa en la tabla 4.

Tabla 4: Configuración Grupo de Estudio

\begin{tabular}{|l|c|c|c|c|}
\hline Población & Universo & Muestra & Edad & Representatividad \\
\hline $\begin{array}{l}\text { Pedagogía Educación } \\
\text { General Básica }\end{array}$ & 442 & 224 & $18-25$ años & $51 \%$ \\
\hline $\begin{array}{l}\text { Escuela Matriz (1) } \\
\begin{array}{c}118 \text { (primer } \\
\text { año } \\
\text { formación) }\end{array}\end{array}$ & 118 & $18-21$ años & $100 \%$ \\
\hline
\end{tabular}

Fuente: elaboración propia

(1) Para la aplicación del ACRA, se tuvo en consideración recopilar el registro de notas que indican el Rendimiento Académico de los estudiantes en las asignaturas de Lenguaje y Comunicación y Matemática, así también como el promedio general de primer año a objeto de llegar a determinar la existencia de alguna relación entre las estrategias de aprendizaje empleadas por los estudiantes, las notas obtenidas en las asignaturas definidas y el rendimiento académico en general.

Para efectuar los análisis estadísticos descriptivos, con posterioridad a la determinación de las estrategias de aprendizaje empleadas por ambas muestras, se utilizaron medidas de tendencia central y dispersión. Además, se empleó la Prueba T de Student, en atención a que dicho estadígrafo permite comparar las medias en un mismo grupo, en este caso permitió determinar la existencia de diferencias entre los diferentes niveles de formación universitaria y el uso de estrategias de aprendizaje.

Por su parte, el carácter correlacional del estudio se vio favorecido por la utilización de la prueba estadística Coeficiente de Correlación de Pearson, en atención a que dicha prueba indica la fuerza y dirección de la relación lineal y proporcional entre dos variables estadísticas, en este caso rendimiento académico y empleo de estrategias de aprendizaje.

Respecto del procedimiento de investigación, cabe mencionar que se inició estableciendo contacto con las instituciones participantes incluidas en la muestra, a quienes formalmente se les invitó a participar dando a conocer los alcances del estudio. Posteriormente, se procedió a aplicar el Test de manera anónima y voluntaria en el tiempo que lo establece dicho instrumento (45 a 60 minutos), en un momento único para cada una 
de las muestras. Posteriormente, se recogió información referida a los rendimientos académicos obtenidos a través de información disponible en sistemas informáticos de la Institución, para el caso de la Escuela Matriz.

\subsection{Descripción del instrumento}

El Test ACRA elaborado por José María Román Sánchez y Sagrario Gallego Rico, docentes de la Universidad de Valladolid se trata de un instrumento constituido por cuatro escalas independientes que evalúan el uso que hacen los estudiantes en las siete técnicas de Adquisición de Información, doce técnicas de Codificación, cuatro de Recuperación y nueve de Apoyo al procesamiento, las cuales se especifican de la siguiente forma:

- La escala de Adquisición de la Información contiene: exploración, subrayado lineal, subrayado idiosincrático, epigrafiado, repaso en voz alta, repaso mental y repaso reiterado.

- La escala de Codificación de Información contiene nemotecnias, relaciones, imágenes, metáforas, aplicaciones, autopreguntas, parafraseado, agrupamientos, secuencias, mapas, diagramas.

- La escala de Recuperación de la Información incluye búsqueda de codificaciones, búsqueda de indicio, planificación de repuestas y respuesta escrita.

- La escala de Apoyo al Procesamiento de la Información incluye: autoconocimiento, automanejo, planificación, autoinstrucción, autocontrol, contradistractoras, interacciones sociales, motivación intrínseca, motivación extrínseca y motivación de escape.

\section{Resultados y Análisis}

Con el objeto de facilitar el análisis de los resultados de las dos investigaciones realizadas, los resultados o hallazgos obtenidos son analizados de manera separada para facilitar su comprensión.

\subsection{Escuela Matriz}

Tal como se ha señalado, la investigación desarrollada busca determinar en este grupo de estudiantes los siguientes aspectos:

- El repertorio de estrategias de aprendizajes empleadas. 
- La relación existente entre el repertorio de estrategias y el rendimiento académico en las asignaturas de Lenguaje y Comunicación y Matemática.

A partir de dichos elementos orientadores se obtuvo lo que se detalla:

a) La Tabla 5 permite sintetizar el repertorio de estrategias de aprendizaje, explicitada a través de los puntajes promedio del valor de respuestas y del puntaje total obtenido mediante la aplicación del Test ACRA.

Tabla 5: Puntaje obtenido en Test ACRA

\begin{tabular}{|c|c|c|}
\hline Variables / escala & $\begin{array}{l}\text { Promedio Puntaje } \\
\text { ACRA }\end{array}$ & $\begin{array}{l}\text { Promedio valor } \\
\text { respuesta * }\end{array}$ \\
\hline $\begin{array}{l}\text { Promedio Estrategias de Adquisición/ } \\
\text { escala I }\end{array}$ & 52.6 & 2,6 \\
\hline $\begin{array}{l}\text { Promedio Estrategias de Codificación/ } \\
\text { escala II }\end{array}$ & 118.68 & 2,6 \\
\hline $\begin{array}{l}\text { Promedio Estrategias } \\
\text { Recuperación/ escala III }\end{array}$ & 51.48 & 2,9 \\
\hline $\begin{array}{l}\text { Promedio Estrategias De Apoyo/ } \\
\text { escala IV }\end{array}$ & 102.55 & 2,9 \\
\hline Puntaje total & 325.31 & 2.75 \\
\hline
\end{tabular}

Fuente: elaboración propia

(*) Las escalas son puntuadas desde el 1 al 4.

Al observar los resultados de los valores obtenidos en las cuatro escalas que mide el Test ACRA, se demuestra que el repertorio empleado por los estudiantes (dicha escuela matriz solo admite varones) es mayor en las estrategias de apoyo y de recuperación. Esto implica que dichos jóvenes posean un importante repertorio para traer la información a la memoria de corto plazo, lo cual además se ve favorecido porque poseen estrategias que le otorgan seguridad en sí mismo. Lo anterior, no deja de ser interesante de considerar puesto que en dicha institución militar lo relacionado con auto confianza, orgullo por pertenecer a una rama militar y el valor de patriotismo, quedarían de manifiesto en una actitud de seguridad en sí mismo.

En contraposición, las Estrategias de Adquisición y de Codificación son las que presentan un bajo promedio de empleo, lo cual resta potencia al valor obtenido en las otras dos escalas (Recuperación y de Apoyo). Esto indicaría que a los estudiantes se les dificulta conectar la información nueva con los conocimientos previos para integrarlos a estructuras de significado más amplias. Teniendo en consideración que 
las estrategias para la búsqueda de la información almacenada se hallan básicamente condicionadas por la organización de los conocimientos en la memoria, entonces se constituye en factor clave poseer un adecuado repertorio, tanto de las Estrategias de Adquisición como de las de Codificación.

En términos generales, los valores obtenidos en las cuatro escalas indican que los estudiantes hacen un uso limitado de todas las estrategias, no existiendo diferencias significativas de ellas.

b) Con el propósito de determinar la existencia de alguna relación entre el repertorio de estrategias y el rendimiento académico en las asignaturas de Lenguaje y Comunicación y Matemática, fue necesaria la aplicación del Coeficiente de Correlación de Pearson con el fin de determinar si habría vinculación entre repertorio y rendimiento.

La tabla 6 permite sintetizar los resultados obtenidos por el grupo de estudiantes de la Escuela Matriz en relación con el rendimiento académico:

Tabla 6: Rendimiento Académico y Desviación Estándar

\begin{tabular}{|l|c|c|}
\hline \multicolumn{1}{|c|}{ Variables } & Media $\left(^{*}\right)$ & Desviación Estándar \\
\hline Lenguaje y Comunicación & 7,52 & 0.76 \\
\hline Matemática & 7,26 & 1.13 \\
\hline Promedio general & 7,65 & 0.67 \\
\hline
\end{tabular}

Fuente: elaboración propia

$\left(^{\star}\right)$ Las calificaciones emplean escala de 1 a 10, donde a partir del 6 es aprobatoria.

Un primer análisis efectuado con el objetivo de determinar relaciones entre las cuatro escalas del Test, permitió determinar una fuerte relación directamente proporcional entre el uso de las Estrategias de Codificación y las de Recuperación de la Información, con un valor correlacional de $\mathrm{R}=0.722$. Es posible señalar entonces que en el grupo evaluado, cuando incrementan el uso de las Estrategias de Codificación, también lo hacen las Estrategias de Recuperación y viceversa. Esto permitiría a través de las técnicas de codificación trasladar la información a la memoria de largo plazo, logrando la comprensión de los conocimientos, de modo que puedan ser recuperados apropiadamente. Por otra parte, las estrategias empleadas para la búsqueda de la 
información se hallan condicionadas por la organización de los conocimientos en la memoria y que son el resultado de las Estrategias de Codificación, por tanto, la calidad de la información recuperada desde la memoria, depende de lo realizado en la fase de codificación.

Donde se observa una menor fuerza en las escalas analizadas se da entre las Estrategias de Adquisición y de Codificación con un valor de correlación $R=0.694$ y entre las Estrategias de Codificación con las Estrategias de Apoyo con un valor de $\mathrm{R}=0.610$. Desde un punto de vista teórico implica que los estudiantes al no contar con un suficiente número de técnicas que favorecen la confianza y seguridad en el proceso de aprendizaje, se les dificulta el proceso de codificación de la información. En relación con este aspecto sería esperable, que los sujetos al presentar una mayor relación entre las distintas estrategias, podrían ver favorecido sus aprendizajes.

Un aspecto interesante de considerar guarda relación con lo observado en aquellos estudiantes que mantienen un mejor promedio general de sus asignaturas, donde tienden a aplicar mayoritariamente Estrategias de Recuperación de la Información. En este sentido, desde un punto de vista teórico, el hecho de que los sujetos posean o manejen un buen repertorio de Estrategias de Recuperación ayudaría a traer a la memoria de corto plazo los conocimientos requeridos en las evaluaciones desarrolladas. No fue posible encontrar en términos generales niveles altos de correlación con las otras escalas.

Tabla 7: Valores de Coeficiente Correlación

\begin{tabular}{|c|c|c|c|c|}
\hline $\begin{array}{l}\text { Escala ACRA } \\
\text { Subsector }\end{array}$ & Adquisición & Codificación & Recuperación & Apoyo \\
\hline $\begin{array}{l}\text { Lenguaje } \\
\text { Comunicación }\end{array}$ & 0.24 & 0.32 & 0.41 & 0.36 \\
\hline Matemática & 0.10 & 0.19 & 0.37 & 0.13 \\
\hline $\begin{array}{l}\text { Rendimiento } \\
\text { General }\end{array}$ & 0.17 & 0.26 & 0.40 & 0.25 \\
\hline
\end{tabular}

Fuente: elaboración propia

La tabla 7 permite visualizar la correlación entre subsector de aprendizaje y ACRA, lo que en términos generales indica que se da una correlación positiva, no alta, entre los dos subsectores y las escalas. Esto implica que a medida que aumentan las calificaciones de uno u otro sector de aprendizaje también lo hace, de manera discreta, el empleo de las estrategias de aprendizaje que mide el ACRA. 
Ciertamente, las evidencias indican que los mejores estudiantes (los que logran un mínimo de un $75 \%$ de rendimiento en las asignaturas de Lenguaje y Matemática) hacen mayor empleo de las técnicas pertenecientes a las distintas escalas, en contraposición con el resto de estudiantes, cuyo empleo es inferior. Los resultados anteriores también coinciden con los encontrados en otras investigaciones donde también se obtiene correlación entre rendimiento y empleo de estrategias; es decir, los estudiantes que tienen mejor rendimiento académico cuentan con un mayor repertorio de estrategias, lo cual les favorecer el aprendizaje y por tanto, obtienen mejor rendimiento académico. Lo anterior, no significa necesariamente que quienes hagan empleo de un mayor repertorio de estrategias alcancen un proceso más profundo de aprendizaje, solo que emplean un mayor repertorio y que además tienen mejor rendimiento en comparación con sus pares.

\subsection{Estudiantes de Formación Pedagógica}

En cuanto a este grupo de estudiantes, la investigación se centró en obtener información respecto de dos aspectos:

- Determinar el repertorio de estrategias de aprendizajes empleadas,

- Determinar la relación existente entre el repertorio de estrategias de aprendizaje empleadas y el avance curricular.

El estudio efectuado arrojó los siguientes antecedentes:

a) En relación con el manejo de un repertorio de Estrategias de Aprendizaje, la Tabla 8 permite determinar por nivel de avance curricular el valor promedio y en general, el valor obtenido por cada escala.

Tabla 8: Resultados Test ACRA por nivel de formación

\begin{tabular}{|l|c|c|c|c|c|}
\hline & Primer Año & Segundo Año & Tercer Año & Cuarto Año & Promedio \\
\hline Adquisición & 2,77 & 2,79 & 2,87 & 2,77 & $\mathbf{2 , 8}$ \\
\hline Codificación & 2,45 & 2,43 & 1,42 & 2,62 & $\mathbf{2 , 2}$ \\
\hline Recuperación & 2,87 & 2,86 & 2,58 & 3,03 & $\mathbf{2 , 8}$ \\
\hline Apoyo & 2,76 & 2,7 & 2,27 & 2,76 & $\mathbf{2 , 6}$ \\
\hline
\end{tabular}

Fuente: elaboración propia

Teniendo en consideración que el Test ACRA puntúa de 1 a 4 el valor de cada técnica perteneciente a cada una de las cuatro escalas, permite señalar que los estudiantes emplean un mayor repertorio en las escalas pertenecientes a Adquisición 
de la Información y Recuperación de la Información, en detrimento de las de Codificación y de Apoyo las cuales son empleadas con menor periodicidad.

Se observó que las escalas referidas a Estrategias de Adquisición de Información y Estrategias de Recuperación de Información, son aquellas que presentan valores más altos (2.8 en promedio), respecto de las otras dos escalas. Lo anterior significa que los estudiantes evaluados hacen uso de un importante número de estrategias que les permiten identificar lo sustancial de la información analizada, lo cual facilitaría que dicho conocimiento lo pudiesen almacenar en la memoria de largo plazo. Sin embargo, la Escala de Codificación, teniendo como base el valor obtenido, indicaría que los jóvenes universitarios emplearían un repertorio restringido de dichas estrategias, lo cual no les asegura agrupar los conocimientos de manera de poder hacer uso de ellos cuando lo requieran.

Las evidencias obtenidas son coincidentes con los planteamientos que realizan Nisbet y Shucksmith (1987) y al cual también hacen alusión los autores del test ACRA (Román y Gallego), en orden a que las estrategias cognitivas de aprendizaje o estrategias de procesamiento, son definidas como secuencias integradas de procedimientos o actividades mentales que se activan con el propósito de facilitar la adquisición, el almacenamiento y/o utilización de la información, de allí entonces que sea interesante investigar respecto de qué procedimientos o actividades mentales emplearía dicha población estudiantil, en contraposición a sus pares que tienen un menor rendimiento en sus actividades de aprendizaje.

Quizá lo más característico del grupo, avalado por lo planteado por Román y Gallego (2001), sería que los procesos de naturaleza metacognitivos, de orética, sociales serían los que contribuirían a alcanzar el pleno potencial de los otros tres tipos de estrategias (adquisición, codificación y recuperación). En este sentido, evidentemente este grupo basa su buen resultado académico (medido a través de sus calificaciones) en cuanto al control de sus emociones, la ansiedad, el sobreponerse a los distractores y sus altas expectativas tanto en la confianza de sus capacidades personales para obtener el título profesional, como también la organización (planificación) y la responsabilidad.

b) Respecto del impacto en las Estrategias de aprendizaje a raíz del avance curricular del Plan de Estudio. 
La tabla 9 permite mostrar los valores encontrados en la aplicación del Test ACRA en cada una de los cuatro niveles de admisión en la carrera en estudio:

Tabla 9: Resultados Test ACRA según avance curricular:

\begin{tabular}{|l|c|c|c|}
\hline Admisión & $\begin{array}{l}\text { Puntaje } \\
\text { Total ACRA }\end{array}$ & \% logro & $\begin{array}{l}\text { Promedio } \\
\text { valor } \\
\text { respuesta * }\end{array}$ \\
\hline Primer año & 316.9 & 66.6 & 2.71 \\
\hline Segundo año & 314.3 & 66.0 & 2.69 \\
\hline Tercer año & 249.2 & 52.4 & 2.28 \\
\hline Cuarto año & 327.5 & 68.8 & 2.79 \\
\hline
\end{tabular}

Fuente: elaboración propia

$\left(^{*}\right)$ Las escalas son puntuadas desde el 1 al 4.

Los valores encontrados, aplicando la $\mathrm{T}$ de Student, indican que en los cuatro grupos investigados (primero a cuarto año de formación) no presentan diferencias significativas. A saber, los resultados obtenidos en la prueba en general considerando los grados de libertad, arrojaron un valor "t" general 7,490728E-15, de lo que se desprende que si partimos de la hipótesis que los valores diferían entre los grupos, ésta debe ser rechazada debido a que el valor obtenido resulta inferior al valor de la tabla, tanto con nivel de confianza de $.05(7,490728 \mathrm{E}-15<1.645)$ como con un nivel de confianza de $0.1(7,490728 \mathrm{E}-15<2.326)$. Esto señalaría que el proceso de formación profesional de cuatro años, no incrementa el empleo de las estrategias de aprendizaje. Esto pudiera implicar aspectos como:

- que estas no se incrementan sólo por el hecho de participar de procesos cognitivos, lo que requeriría cierta intencionalidad.

- que los docentes responsables de formar profesionales no estarían preocupados por desarrollar estrategias de aprendizaje en sus estudiantes.

- que los docentes y estudiantes desconocen que para que se produzca incremento en el repertorio de las estrategias de aprendizaje hay que sostener un trabajo sistemático que implicaría no solo su empleo sino que también se traduzca en beneficios concretos; por ejemplo, mejor rendimiento académico.

\section{Conclusión}

A la luz de las evidencias obtenidas en ambas investigaciones se ha podido obtener información que permite comprender y reflexionar en torno a la implementación de mejoras en la formación - tanto a nivel de formación técnica en una escuela matriz de las Fuerzas 
Armadas como en la formación de profesionales de la educación, con el objetivo de favorecer mejores aprendizajes en estudiantes de educación superior. A saber, a través de la aplicación del Test ACRA, se ha podido demostrar la correlación directa que se da entre estudiantes que poseen buen rendimiento académico a nivel general con el mayor empleo de las estrategias de aprendizaje. En otras palabras, estos estudiantes cuentan con un mayor repertorio de técnicas, lo que les favorece el aprender.

En este sentido y a partir de las evidencias obtenidas en ambos segmentos de estudiantes, se puede pensar en la conveniencia de potenciar las estrategias de aprendizaje en la población estudiantil, en especial, en estudiantes que poseen recursos limitados lo cual pudiera redundar en una mejora en los aprendizajes y de esa manera manifestar coherencia con el nuevo tipo de curriculum declarado para la formación de ciudadanos del siglo XXI. Al respecto, entonces, si de manera explícita los académicos de educación superior mostraran énfasis en incentivar y promover el empleo de un espectro más amplio de técnicas asociadas a estrategias de aprendizaje, tal vez se impacte en dicha formación.

Coincidentemente con el planteamiento de Leahey y Harris (2003), en relación con la conveniencia de poseer conocimiento, consciencia y control respecto de los procesos de pensamiento, la población estudiantil que alcanza un mejor rendimiento lograría un mejor control de dichos procesos, adecuando las técnicas para así responder de manera eficiente a las tareas.

Estudios realizados por Fernández, Martínez-Conde, y Melipillán (2009) también encuentran que estudiantes universitarios que suelen emplear estrategias de aprendizaje más complejas, presentan significativamente mejor rendimiento académico, en concomitancia, además, con mayores niveles de autoestima general, académica y familiar. Igualmente, dicho planteamiento es coincidente con los estudios realizados por Martínez y Galán (2000), con el fin de encontrar una correlación positiva entre las estrategias de aprendizaje y rendimiento académico mediado por lo motivacional. De igual manera, investigaciones realizadas en estudiantes del área de la salud evidencian la misma relación (Herrera, 2014). Por tanto, un desafío o consideración en cualquier proceso de formación profesional debería valorar el impacto positivo que generaría en los estudiantes si ellos están motivados por el proceso que están vivenciando.

En este sentido, lo investigado permite reflexionar y continuar debatiendo en relación con el planteamiento que docentes suelen hacerse en función de explicarse por qué razón la población estudiantil no alcanza los aprendizajes por ellos propuestos, lo cual se transforma 
en un diálogo de sordos, dado que estos, a su vez, siguen responsabilizando a la mala calidad de la enseñanza que reciben de sus profesores como la variable clave en la baja calidad de los aprendizaje alcanzados (Marchesi y Martín, 2000).

Un segundo aspecto en coherencia con los resultados obtenidos, indicó que las estrategias de aprendizaje no se incrementan conforme avanza el plan de formación en profesores primarios. Al respecto, es posible señalar que quien posee un mejor nivel en el empleo de estrategias de aprendizaje es también exitoso en sus estudios, dada la relación directa existente entre poseer un mayor repertorio de técnicas que favorecen los aprendizajes. También es posible indicar que si no existe intencionalidad en el proceso de formación por incentivar o ampliar el repertorio de estrategias en los estudiantes, estas no emergen de manera espontánea si los procesos evaluativos siguen favoreciendo la memorización o repetición de contenidos. En este sentido, y siguiendo el planteamiento de evaluación comprensiva que hace Santos Guerra (2001), esta busca jerarquizar o ranquear acorde a sus rendimientos con un enfoque evaluativo eminentemente psicométrico y no con un fin que favorezca la meta-cognición de los procesos. Igualmente, esto es coincidente con lo declarado en el estudio efectuado por Díaz-Barriga y Hernández (2005), donde se encontró congruencia entre las actividades estratégicas con las actividades evaluativas y las condiciones que puedan afectar el uso espontáneo de las estrategias.

En este contexto se coincide con el planteamiento que realizan Díaz-Barriga y Hernández 2005 en relación con la necesidad de que el profesor enseñe a sus estudiantes como aprender de manera efectiva y eficiente sin importar el nivel escolar como los contenidos.

Teniendo como base lo señalado anteriormente, las estrategias de aprendizaje deben ser entendidas como un repertorio, un conjunto de habilidades que se emplean con un fin y objetivo preciso por lo cual, y en atención a dicha particularidad, no basta con saber que existen y que tienen tal o cual impacto, sino que, deben ser empleadas para controlar y regular otras habilidades más específicas. Estas son catalogadas como habilidades de orden elevado que no pueden ser entendidas como secuencias, conjunto de habilidades, reglas, ni menos, como sólo hábitos, sino que al involucrar procesos como la planificación y la comprobación requieren un proceso activo por quien las emplee con el propósito de alcanzar los procesos intelectivos requeridos en las circunstancias del proceso educativo llevado a cabo. 
Asociadas con el concepto de competencias, entendidas estas como la movilización de un conjunto de recursos, sean de conocimiento, habilidades y actitudinales para actuar de manera competente, es viable señalar que las estrategias de aprendizaje son una serie de competencias necesarias o útiles para el aprendizaje efectivo y la retención de la información y su uso posterior. Dichas competencias incluyen estrategias cognitivas y de procesamiento de la información como estrategias para organizar y elaborar la información entrante y hacerla más significativa, entre ellas: estrategias de estudio como la toma de notas o la preparación del examen, y estrategias de apoyo, es decir, técnicas para organizar el tiempo, superar la ansiedad de ejecución y dirigir la atención a la tarea de aprendizaje.

En síntesis, conocer cuáles estrategias de aprendizaje emplean estudiantes de Educación Superior no sólo sirve para obtener su caracterización, sino que por sobre todo, apunta a comprender qué nivel de profundidad alcanzan; por una parte, en los procesos intelectivos que desarrollan y por otra, reflexionar en torno a qué acciones efectúan dentro del proceso de enseñanza o dentro del Plan de Estudio, para que todo el cuerpo docente promueva y potencie un mayor número de estrategias de aprendizaje y que ellas redunden en los niveles cognitivos que se desean alcanzar en el momento de verificar el dominio del conocimiento o más aún, en la aplicación que se haga de dicha información por parte de la población estudiantil.

\section{Referencias}

Arancibia, Violeta, Herrera, Paulina y Strasser, Katherine. (2005). Manual de Psicología Educacional. Santiago, Chile: Ediciones Universidad Católica de Chile.

Ausubel, David, Novak, Joseph y Hanesian, Helen. (1997). Psicología Educativa, un punto de vista cognoscitivo. México: Editorial Trillas.

Ballester, Rafael. (2002). Habilidades sociales: evaluación y tratamiento. Madrid: Síntesis.

Beltrán, Jesús. (1998). Procesos, Estrategias y Técnicas de Aprendizaje. Madrid: Síntesis.

Bruner, Jerome. (2004). Desarrollo Cognitivo y Educación. Madrid: Morata.

Carbonero, Miguel Ángel, Román, José María y Ferrer, Marisela. (2013). Programa para "aprender estratégicamente" con estudiantes universitarios: Diseño y validación. Anales de psicología, 29(3), 876-885.

Coll, César, Martín, Elena y Mauri, Teresa. (2007). El constructivismo en el Aula. Barcelona: Graó. 
Cook, Linda y Mayer, Richard. (1983). Reading Strategies Training for Meaningful Learning from Prose. In M. Pressley et al. (eds.), Cognitive Strategy Research (pp. 87-131). New York: Springer-Verlag.

Correa, María Elena, Castro, Fancy y Lira, Hugo. (2004). Estudio descriptivo de las estrategias cognitivas y metacognitivas de los alumnos y alumnas de primer año de pedagogía en enseñanza media de la Universidad del Bío-Bío. Revista Theoria, 13, 103-110.

Díaz-Barriga, Frida y Hernández, Gerardo. (2005). Estrategias Docentes para un Aprendizaje Significativo. México: McGraw- Hill.

Fernández, Olga, Martínez-Conde, Macarena y Melipillán, Roberto. (2009). Estrategias de aprendizaje y autoestima. Su relación con la permanencia y deserción universitaria. Revista Estudios Pedagógicos, 35(1), 27-45.

Flavell, John. (2000). El Desarrollo Cognitivo. Madrid: Visor.

Freire, Paulo. (1999). Pedagogía del oprimido. México: Siglo Veintiuno Editores.

Henson, Kenneth y Eller, Ben. (2000). Psicología Educativa para la Enseñanza Eficaz. México: Thomson Editores S.A. de C.V.

Hernández, Roberto, Fernández, Carlos y Baptista, Pilar. (2010). Metodología de la Investigación. México: McGraw-Hill.

Herrera, Francisca. (2014). Motivación, estrategias de aprendizaje y rendimiento académico, en alumnos de Nutrición y dietética de la Universidad del Desarrollo. Revista Educación en Ciencias de la Salud, 11(1), 38-46.

Leahey, Thomas y Harris, Richard. (2003). Aprendizaje y Cognición. Madrid: Pearson Educación, S.A.

Marchesi, Álvaro y Martín Elena. (2000). Calidad de la Enseñanza en Tiempos de Cambio. Madrid: Alianza S.A.

Martínez, Reynaldo y Galán, Ferrán. (2000). Estrategias de Aprendizaje, motivación y rendimiento académico en alumnos universitarios. Revista Española de Orientación y Psicopedagogía, 11(19), 35-50.

Mayer, Richard. (2002). Psicología de la Educación. El aprendizaje en las Áreas del Conocimiento. Madrid: Pearson Educación, S.A.

Mayor, Juan, Suengas, Aurora y González, Javier. (1995). Estrategias Metacognitivas. Madrid: Síntesis S.A.

Monereo, Carles, Castelló, Monserrat y Clariana, Mercé. (2006). Estrategias de Enseñanza y Aprendizaje. España: Graó.

Nisbet, John y Shucksmith, Janet. (1987). Estrategias de Aprendizaje. Madrid: Santillana. 
Pozo, Juan y Monereo, Carles. (2000). El aprendizaje Estratégico. Madrid: Santillana S.A.

Pozo, Juan y Pérez, María. (2009). Psicología del Aprendizaje Universitario: La Formación en Competencias. Madrid: Morata S.L.

Pozo, Juan. (2005). Aprendices y Maestros. Madrid: Alianza S.A.

Puente, Anibal. (1997). Estilos de Aprendizaje y Enseñanza. Madrid: España.

Román, José María y Gallego, Sagrario. (2001). ACRA, Escala de Estrategias de Aprendizaje. Madrid: TEA Ediciones, S.A.

Rué, Joan. (2009). El Aprendizaje Autónomo en Educación Superior. España: Narcea, S. A. de Ediciones.

Santos Guerra, Miguel Ángel. (2001). Enseñar o el oficio de aprender: organización escolar y desarrollo profesional. Santa Fe: Homo Sapiens.

Vieytes, Rut. (2004). Metodología de la investigación en organizaciones, mercado y sociedad: epistemología y técnicas. Buenos Aires: De la Ciencia. 